This item was submitted to Loughborough's Research Repository by the author.

Items in Figshare are protected by copyright, with all rights reserved, unless otherwise indicated.

\title{
Quantifying elbow extension and elbow hyperextension in cricket bowling: a case study of Jenny Gunn
}

PLEASE CITE THE PUBLISHED VERSION

http://dx.doi.org/10.1080/02640414.2012.682082

PUBLISHER

(c) Taylor \& Francis

VERSION

AM (Accepted Manuscript)

LICENCE

CC BY-NC-ND 4.0

\section{REPOSITORY RECORD}

King, Mark A., and Maurice R. Yeadon. 2019. "Quantifying Elbow Extension and Elbow Hyperextension in Cricket Bowling: A Case Study of Jenny Gunn”. figshare. https://hdl.handle.net/2134/10018. 
This item was submitted to Loughborough's Institutional Repository (https://dspace.lboro.ac.uk/) by the author and is made available under the following Creative Commons Licence conditions.

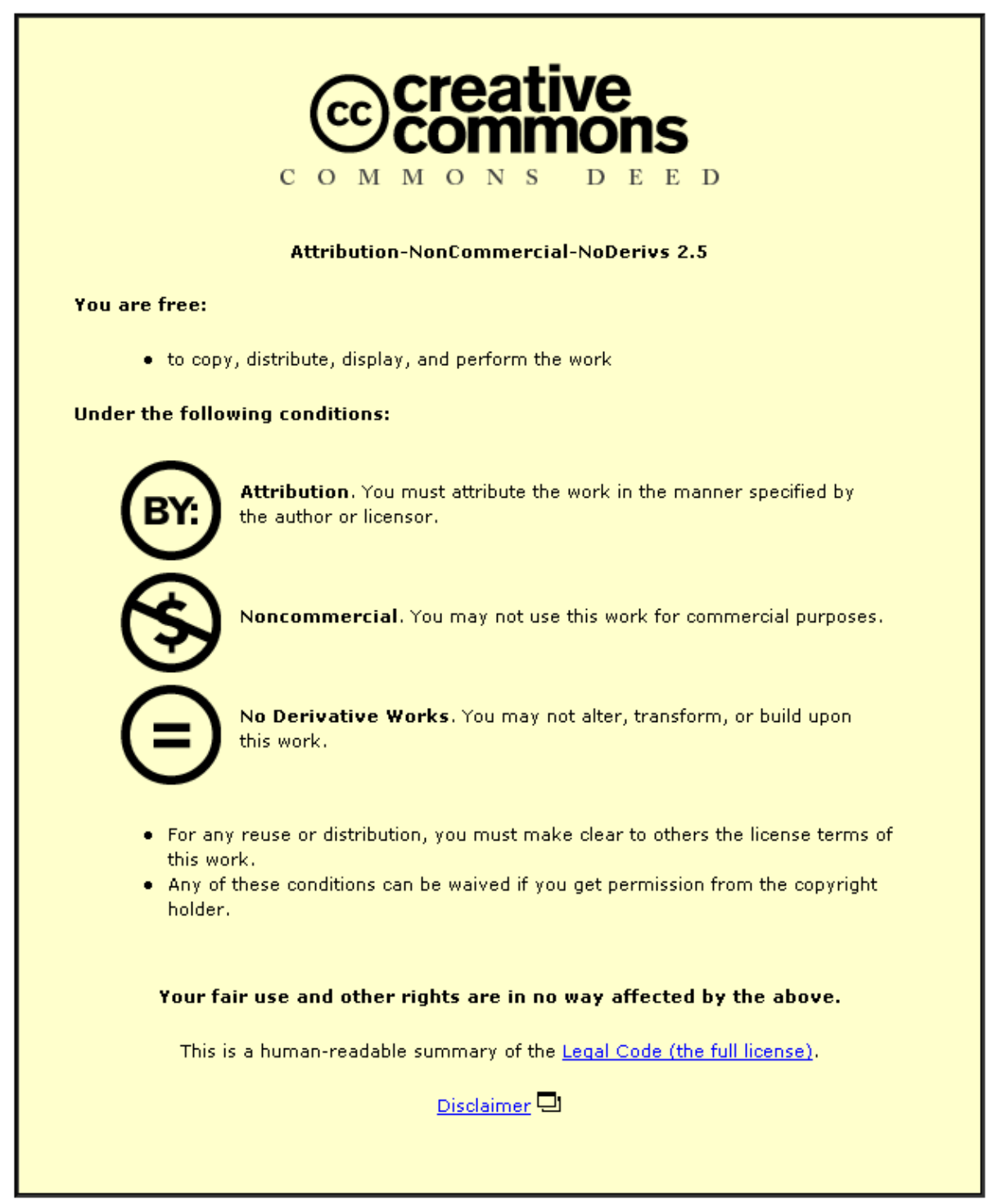

For the full text of this licence, please go to: http://creativecommons.org/licenses/by-nc-nd/2.5/ 


\title{
Quantifying elbow extension and elbow hyperextension in cricket bowling: A case study of Jenny Gunn
}

\author{
Mark A. King and Maurice R. Yeadon \\ School of Sport, Exercise and Health Sciences, Loughborough University, UK.
}

\begin{abstract}
In this study a method for determining elbow extension and elbow abduction for a cricket bowling delivery was developed and assessed for Jenny Gunn who has hypermobility in both elbows and whose bowling action has been repeatedly queried by umpires. Bowling is a dynamic activity which is assessed visually in real time in a cricket match by an umpire. When the legality of a bowler's action is questioned by an umpire a quantitative analysis is undertaken using a marker based motion analysis system. This method of quantifying elbow extension should agree with a visual assessment of when the arm is "straight" and should minimise the effects of marker movement. A set of six markers on the bowling arm were used to calculate elbow angles. Differences of up to $1^{\circ}$ for elbow extension and up to $2^{\circ}$ for elbow abduction were found when angles calculated from the marker set for static straight arm trials were compared with measurements taken by a chartered sports physiotherapist. In addition comparison of elbow extension angles at ball release calculated from the markers during bowling trials with those measured from high speed video also showed good agreement with mean differences of $0^{\circ} \pm 2^{\circ}$.
\end{abstract}

\section{Introduction}

The topic of whether a bowler is bowling legally or not is a contentious issue that causes much debate and confusion. The current procedure is that if an umpire is of the opinion that a player is bowling with an action which contravenes Law 24 of the International Cricket Council (ICC) rules then an independent analysis of their bowling action will be carried out by an approved human movement specialist under laboratory conditions (ICC, 2009).

In recent years the most high profile player to be "called" is Muttiah Muralitharan whose bowling action was shown to be legal (Lloyd et al., 2000; Bartlett, 2003) but unusual due to a fixed-flexion deformity. More recently Jenny Gunn (a member of the England Women's cricket team) has had her bowling action repeatedly questioned. Indeed, in February 2009, just a few days before the start of the Cricket World Cup, Miss Gunn was suspended from bowling in domestic matches in Australia after her action was deemed illegal, although an investigation by the ICC subsequently gave her the all-clear to play in the World Cup.

The International Cricket Council regulations (ICC, 2009) currently permit the elbow to extend by up to $15^{\circ}$ during the period between the upper arm being horizontal and the instant of ball release (not including any elbow abduction angle changes or elbow hyperextension). Consequently a method is needed that will measure accurately the elbow extension angle during a delivery, and will identify when the arm is "straight" so that elbow hyperextension can be identified accurately. Ideally an on-field marker-less method for calculating elbow extension would be used, but this is currently not possible. Video analysis can be used to calculate the elbow extension angle at a specific instant during the bowling action when the elbow axis is approximately parallel to the video camera axis. However, it is not possible to calculate elbow extension angle time histories from a fixed camera location as the 
orientation of the elbow axis changes throughout the bowling action (Aginsky and Noakes, 2010; Portus et al., 2006). As a consequence a laboratory marker based method is used. The number of markers should be small so as to interfere minimally with the ability of the bowler to make typical deliveries (Lloyd et al., 2000). The time needed to position the markers and to calibrate the elbow axis direction should also be minimised.

The current ICC testing protocols (ICC, 2009) for establishing whether a bowler is extending his/her arm legally leaves some flexibility in interpretation of where motion analysis markers should be placed, how to identify a straight arm and exactly how the elbow flexion/extension axis should be defined, with the current method based upon a protocol developed by the University of Western Australia (Lloyd, et al., 2000). This protocol used three markers located centrally on the upper arm and two markers located centrally on the forearm in order to determine the relative orientation of the two arm segments. This choice of marker placement was made since markers placed over joints suffer from skin movement as the joint angle changes (Cappozo et al., 1996). While skin movement may be the over-riding consideration in clinical gait studies, there can be substantial soft tissue movement in dynamic sports activities (Roosen et al., 2009) which can produce movement artefact in markers located centrally on a segment. This effect may be responsible for the University of Western Australia (UWA) Group changing from central marker placement in favour of small marker triads positioned on the upper arm near the shoulder and elbow (Zhang et al., 2011). This paper attempts to remove the effect of soft tissue artefact using filtering but concludes that the most realistic way to reduce soft tissue artefact is to position markers on areas least affected by soft tissue movement. As a consequence there is a case for a fresh look at marker placement since a pair of markers placed medially and laterally across the elbow are less likely to be affected by soft tissue movement and skin movement will be small if the elbow angle changes are small.

It is crucial to know how accurate calculated elbow angles are. Angles calculated from markers placed on a mechanical bowling arm had an error of $0.6^{\circ}$ (Elliott et al., 2007). While this gives some confidence in the methods used, there remains the problem of soft tissue movement and skin movement in human bowling. As a consequence there needs to be an assessment of the accuracy of marker based methods of angle calculations in bowling.

Identifying exactly when the bowling arm is in a "straight" (extended) position is critical for some bowlers (e.g. Jenny Gunn as she has extension hypermobility in both elbows). At present this issue has not been addressed with it being assumed that the bowling arm is straight when the wrist joint centre lies in the plane defined by the long axis of the upper arm and the elbow axis. The problem with this approach is that it makes no specific reference to when the arm is visually in a straight position and is implemented by defining the upper arm axis in terms of the shoulder and elbow joint centres. Judgements made by umpires are based on a visual assessment of arm configuration. As a consequence a method is required that defines a straight arm to be when the arm is visually in a straight arm position so that an elbow extension angle of $180^{\circ}$ corresponds to a straight arm.

The specific definition of the elbow flexion/extension axis may affect the amount of elbow extension for a specific bowling trial. In the current ICC testing protocols (ICC, 2009) reference is made to Lloyd et al. (2000) where the elbow flexion/extension axis is defined to be perpendicular to the longitudinal axis of the 
upper arm in the frontal plane. Others have defined the axis as the line joining the medial and lateral condyles at the elbow in the frontal plane (Alderson et al., 2004) and more recently a functional axis has been used (Chin et al., 2009; Chin et al., 2010). A functional method based upon movement data that gives an accurate estimate of the flexion/extension axis and takes into account an individual bowler's specific joint motion is probably most appropriate and desirable.

An accurate determination of the instants when the upper arm is horizontal and when the ball is released is crucial to the calculation of the amount of elbow extension for a specific delivery. These two instants may not occur at the time intervals that data is captured (current protocols specify a data capture frequency of $250 \mathrm{~Hz}$ ) and so an objective method is needed to identify when these instants occur, together with a method for interpolating between images so that errors in the calculated elbow extension angles at the start and end of the period of interest are minimised. In addition the current regulations state that displacement data should be smoothed. As angles are required for the bowling analysis and since modern motion analysis systems provide accurate movement data, the added complication of smoothing may no longer be necessary.

The aim of this study was to develop an appropriate method for determining the amount of elbow extension/hyperextension and elbow abduction for a bowling delivery, to apply the method to Jenny Gunn who has hypermobility in both elbows, and to quantify the accuracy of the calculated angles.

\section{Methods}

Data was collected on the participant Jenny Gunn during two data collection sessions 18 months apart with the first session in April 2007 and the second in September 2009 (before and after the Cricket World Cup in February 2009). Each session consisted of an anthropometric assessment (carried out by a chartered sports physiotherapist), followed by a range of calibration trials and six deliveries of each type that the participant could bowl (Table 1). For each trial six markers attached to the bowler's arm were tracked using a motion analysis system. In addition high speed video $(300 \mathrm{~Hz})$ of the September 2009 testing was recorded from rear and side views.

All data were collected in the indoor practice facility at the England and Wales Cricket Board National Cricket Centre at Loughborough University. This venue allowed the participant to bowl with her normal length run-up on a standard size artificial cricket pitch with each delivery recorded using an 18 camera Vicon Motion Analysis System (OMG Plc, Oxford, UK) operating at $250 \mathrm{~Hz}$ (April 2007) and $300 \mathrm{~Hz}$ (September 2009). Cameras were positioned around the bowling crease to cover a 7 $\mathrm{m} \times 3 \mathrm{~m} \times 3 \mathrm{~m}$ volume which was wand calibrated prior to data collection. The participant was deemed fit to bowl by an England cricket physiotherapist and had bowled three times per week, on average, in either practice sessions or matches during the current season. The testing procedures were explained to the subject in accordance with Loughborough University ethical guidelines and informed consent was obtained for the data collection procedures, use of her name and subsequent use of images. A chartered sports physiotherapist used a manual goniometer to measure elbow abduction (carry angle) with the arm straight and maximum elbow hyperextension.

Six, $14 \mathrm{~mm}$ diameter, spherical reflective markers were attached to the bowling arm in three pairs using aerosol sports adhesive and double-sided tape 
along with a piece of reflective tape $(\approx 1.5 \mathrm{~cm}$ square $)$ attached to one side of the cricket ball. At the wrist the pair of markers were positioned near the styloid processes such that the midpoint of the pair of markers lay on the midline of the lower arm (Figure 1a). At the elbow the pair of markers were positioned vertically above the medial and lateral elbow epicondyle bony landmarks (when the arm was horizontal and the palm of the hand was facing upwards) so that the midpoint of the pair of markers lay on the midlines of the upper arm and lower arm (Figure 1b). At the shoulder the pair of markers were positioned (anterior and posterior to the shoulder) with the arm overhead so that the line joining these markers intersected the midline of the upper arm (Figure 1c).

\section{(a)}
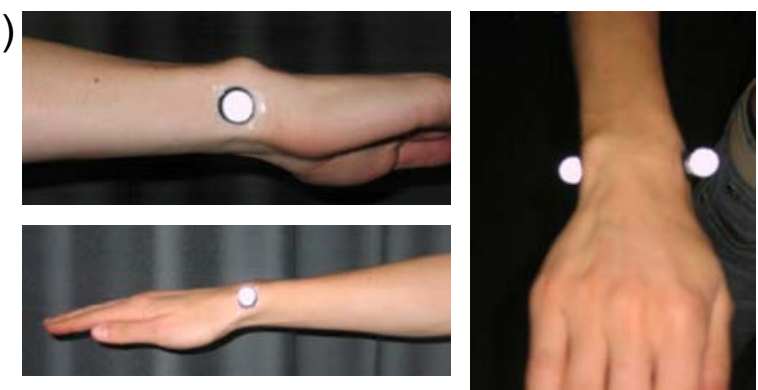

(b)
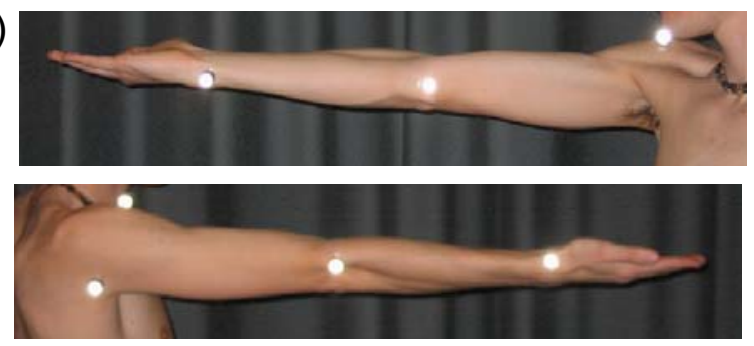

(c)

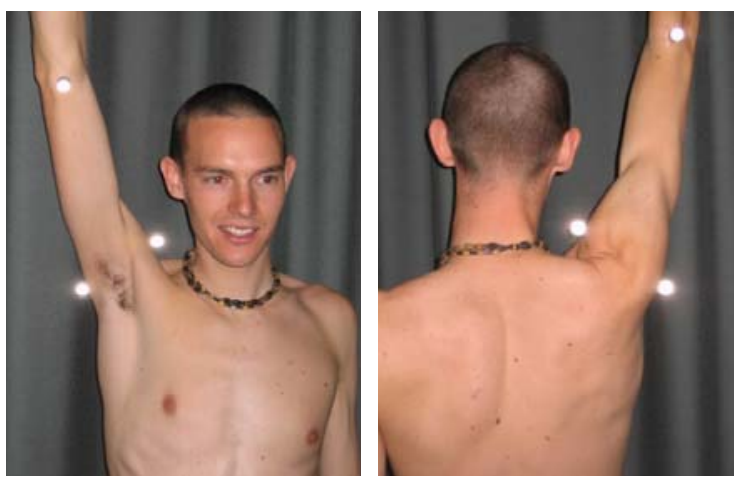

Figure 1. Reflective marker locations.

A range of calibration trials were recorded prior to the subject bowling six of each type of delivery that the subject was comfortable bowling (Table 1). These comprised: three static "straight arm trials" where the bowling arm was visually placed overhead in a straight position by a chartered sports physiotherapist (midlines of the upper arm and lower arm lying in a plane through the elbow markers (Figure 2); an "elbow flexion trial" where from a straight arm position overhead the arm was flexed at the elbow while keeping the upper arm stationary and then extended back to the straight arm position; and a static "ball release trial" where the ball was held touching the end of the finger tips of the bowling hand. In addition for the April 2007 data collection three static forced overhead "hyperextension trials" were recorded, in 
which the physiotherapist held the bowling arm overhead in a 'maximal' hyperextended position.

Table 1. Data collection details

\begin{tabular}{lcc}
\hline & \multicolumn{2}{c}{ number of trials } \\
& April 2007 & September 2009 \\
\hline straight arm & 3 & 3 \\
hyperextended & 3 & - \\
elbow flexion & 1 & 1 \\
ball release & 1 & 1 \\
\hline length & 6 & 12 \\
yorker & 6 & 6 \\
bouncer & - & 6 \\
slower & 6 & - \\
\hline
\end{tabular}

Note: the 12 length deliveries were split into 6 deliveries using her 2007 bowling action and 6 deliveries using a modified bowling action.

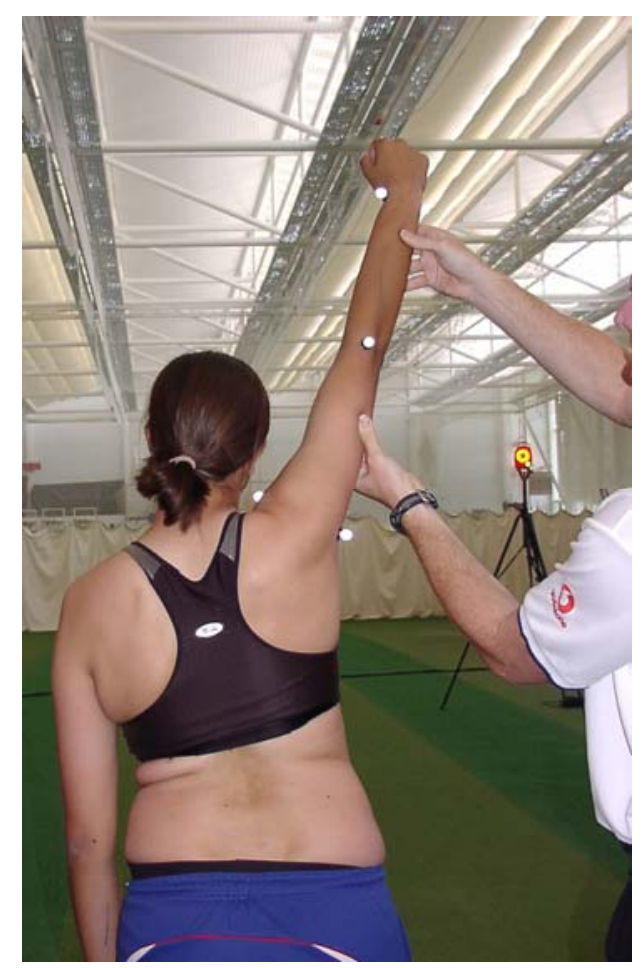

Figure 2. Static "straight arm trial".

All trials were manually labelled and initially processed using the Vicon Workstation and BodyBuilder software with all trials tracked without any marker loss. The effect of smoothing the displacement data was investigated using a fourth-order low-pass Butterworth filter (double-pass) with cut-off frequencies of $10 \mathrm{~Hz}, 20 \mathrm{~Hz}, 30$ $\mathrm{Hz}$ and $40 \mathrm{~Hz}$ for all marker trajectories. The lower arm endpoint $\mathrm{W}$ was defined as the midpoint of the pair of markers at the wrist; the elbow centre $E$ was defined as the midpoint of the pair of markers at the elbow; the upper arm endpoint $S$ was defined as a weighted average of the pair of markers placed around the shoulder joint (Figure 3). A weighting of 40:60 was used with the point $S$ being closer to the 
posterior shoulder marker. This weighting was determined from photographs of the bowler with the markers attached and her arm overhead, assuming that $S$ lay on the midline of the upper arm. These definitions were consistent with having an elbow extension angle of $180^{\circ}$ when the midlines of upper and lower arm and the elbow axis all lie in one plane. The instant of upper arm horizontal was defined as the first image during the delivery when the vertical location of the elbow joint centre was higher than the upper arm endpoint, while the instant of ball release was defined as the first image where the distance between the ball marker and the lower arm endpoint was greater than the measured distance from the static calibration ball release trial: $240 \mathrm{~mm}$ (April 2007) and $237 \mathrm{~mm}$ (September 2009). Linear interpolation was used to identify between images the instants of upper arm horizontal and ball release in order to compare with the above definition. The 3D coordinates of the reflective tape on the ball were used to calculate ball release velocity as the average resultant velocity calculated over the first five frames in flight from the 3D coordinate data.

The lower arm endpoint W, elbow centre $\mathrm{E}$, upper arm endpoint $\mathrm{S}$, and the pair of markers $\left(E_{L}\right.$ and $\left.E_{M}\right)$ across the elbow for two positions of the arm (straight and flexed to $90^{\circ}$ ) from the elbow flexion calibration trial were used to define a functional elbow axis $E_{\text {axis. }}$. $E_{\text {axis }}$ was defined relative to the plane $S \_E_{M} E_{L}$ such that the lower arm folds on top of the upper arm when fully flexed (Figure $3 a$ and $3 b$ ). The direction of $\mathrm{E}_{\text {axis }}$ was determined using the condition that it made equal angles with $\mathrm{EW}_{1}, \mathrm{EW}_{2}, \mathrm{EW}^{\prime}$ so that the dot products with these three vectors were equal. The extension angle was defined as the angle between the planes $S_{-} E_{\text {axis }}$ and $W E_{\text {axis. }}$. The elbow abduction angle (or carry angle) was defined as: $180^{\circ}-W E \_E_{\text {axis }}$ - SE_E

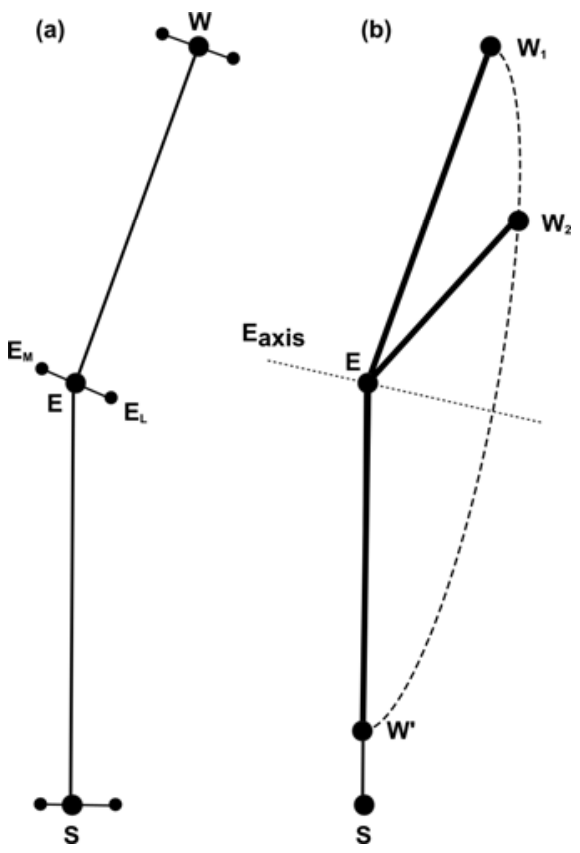

Figure 3. (a) Shoulder, elbow and wrist markers, (b) elbow axis calculation.

Note: $W_{1}$ and $W_{2}$ are two recorded positions of the wrist from the elbow flexion calibration trial for extension angles of approximately $180^{\circ}$ and $90^{\circ} . \mathrm{W}^{\prime}$ is an assumed point on the upper arm midline when the lower arm is fully folded onto the upper arm. $E_{\text {axis }}$ may lie outside of each of the planes $S_{-} E_{M} E_{L}$ and $W_{-} E_{M} E_{L}$ 
The elbow axis $E_{\text {axis }}$ (as determined above) was held in the upper arm reference plane $S_{-} E_{M} E_{L}$ for the determination of angles from the marker motions. As a consequence the angle between $E_{\text {axis }}$ and $E S$ remained constant whereas the angle between $\mathrm{E}_{\text {axis }}$ and $\mathrm{EW}$ could change. Thus while it might be expected that the abduction angle would remain constant in the above calibration trial, there might be additional forced abduction in a bowling trial.

Elbow extension angles and elbow abduction angles were calculated for the arm calibration trials using the marker locations and compared with the angles measured using a manual goniometer by a chartered sports physiotherapist. Elbow extension angles were calculated for the instants of upper arm horizontal, ball release and the most flexed/most extended elbow angles between the upper arm being horizontal and ball release for each bowling trial. The amount of elbow extension for each trial was then calculated as the most extended elbow extension angle (up to $180^{\circ}$ ) minus the preceding minimum elbow extension angle.

Elbow extension angles were measured from the side camera high speed video recordings at ball release (September 2009; three trials of each delivery type bowled) since the elbow axis was approximately parallel to the side camera axis at ball release and so the observed angle between the midlines of the upper arm and lower arm could be measured (e.g. Figure 7) and compared with the elbow extension angles calculated from the marker locations.

\section{Results}

Static anthropometric measurements by a chartered sports physiotherapist found that Miss Gunn had extension hypermobility in both elbows (Figure 4) with a static forced elbow hyperextension angle of $194^{\circ}\left(14^{\circ}\right.$ of hyperextension) and elbow abduction angle (carry angle) of $10^{\circ}$ for her bowling arm.

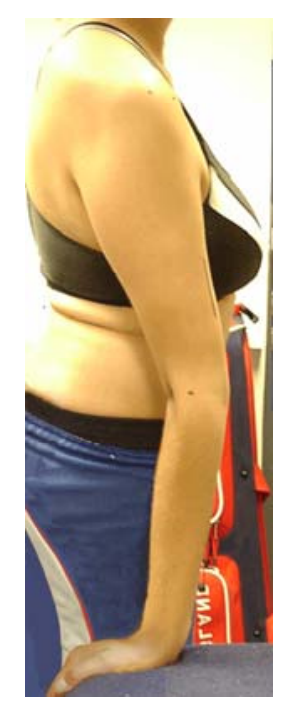

Figure 4. Elbow hypermobility with a large elbow hyperextension angle.

The elbow extension angles calculated from the three visually identified "straight arm calibration trials" were all within $1^{\circ}$ of $180^{\circ}$ (April 2007: $180.4^{\circ} \pm 0.5^{\circ}$; September 2009: $180.1^{\circ} \pm 0.1^{\circ}$ ) along with elbow abduction angles of $10.7^{\circ} \pm 0.4^{\circ}$ (April 2007) and $8.6^{\circ} \pm 0.8^{\circ}$ (September 2009). The three forced overhead "hyperextension trials" resulted in calculated elbow extension angles of $194.0^{\circ} \pm 0.1^{\circ}$ 
(Table 2). In the "elbow flexion calibration trials" the mean abduction angles were $9.5^{\circ} \pm 0.7^{\circ}$ (April 2007) and $11.0^{\circ} \pm 1.1^{\circ}$ (September 2009) for elbow extension angles greater than $150^{\circ}$. All trials were tracked without marker loss.

Table 2. Elbow angles in straight and hyperextension trials, ${ }^{1} 2007$ data, ${ }^{2} 2009$ data

\begin{tabular}{ccc}
\hline & extension & abduction \\
\hline ' straight & $180^{\circ}$ & $10^{\circ}$ \\
& $181^{\circ}$ & $11^{\circ}$ \\
& $180^{\circ}$ & $11^{\circ}$ \\
\hline mean (sd) & $180^{\circ}\left(0.5^{\circ}\right)$ & $11^{\circ}\left(0.4^{\circ}\right)$ \\
\hline${ }^{1}$ hyperextended & $194^{\circ}$ & $14^{\circ}$ \\
& $194^{\circ}$ & $14^{\circ}$ \\
& $194^{\circ}$ & $14^{\circ}$ \\
\hline mean (sd) & $194^{\circ}\left(0.1^{\circ}\right)$ & $14^{\circ}\left(0.3^{\circ}\right)$ \\
\hline${ }^{2}$ straight & $180^{\circ}$ & $8^{\circ}$ \\
& $180^{\circ}$ & $9^{\circ}$ \\
& $180^{\circ}$ & $9^{\circ}$ \\
\hline mean (sd $)$ & $180^{\circ}\left(0.1^{\circ}\right)$ & $9^{\circ}\left(0.8^{\circ}\right)$ \\
\hline
\end{tabular}

All bowling trials in both data collection sessions showed similar characteristics with the bowling arm being close to straight at upper arm horizontal. In every trial the bowling arm then became hyperextended before returning to a relatively straight position at ball release (Figure 5, Figure 6, Table 3). The slower deliveries in the April 2007 data collection were the exception where the elbow was hyperextended throughout the period upper arm horizontal to ball release (Figure 6, Table 3). The range of mean elbow extension from most flexed to most extended (ignoring hyperextension) for the different delivery types was $0.0^{\circ} \pm 0.1^{\circ}$ to $5.6^{\circ} \pm 0.7^{\circ}$ across both data collection sessions (Table 3 ) with a maximum elbow extension of $8.4^{\circ}$ for a bouncer bowled in the September 2009 data collection. The elbow abduction angle time histories showed the same trend for all bowling trials in both data collection sessions (Figure 6, Table 4). The elbow abduction angles at upper arm horizontal (mean $9^{\circ} \pm 3^{\circ}$ ) were close to the statically measured $10^{\circ}$ of abduction. Midway between upper arm horizontal and ball release the bowling arm was forced into a more abducted position (mean $26^{\circ} \pm 2^{\circ}$ ) before returning to a less extreme position by ball release (mean $\left.16^{\circ} \pm 1^{\circ}\right)$. Maximum hyperextension ranged from $15^{\circ}$ to $20^{\circ}$. Estimating the elbow extension angle at ball release (e.g. Figure 7) for three trials of each bowling type from high speed video recordings (side camera position, September 2009 bowling trials) showed good agreement with the angle calculated from the markers with a mean difference of $0^{\circ} \pm 2^{\circ}$ (Table 5). 
Table 3. Elbow extension angles during the bowling trials, ${ }^{1} 2007$ data, ${ }^{2} 2009$ data; mean (sd)

\begin{tabular}{lcccccc}
\hline & ball & \multicolumn{3}{c}{ elbow extension angle $\left(^{\circ}\right)$} & elbow \\
& $\begin{array}{c}\text { speed } \\
(\mathrm{mph})\end{array}$ & $\begin{array}{c}\text { upper arm } \\
\text { horizontal }\end{array}$ & $\begin{array}{c}\text { maximum } \\
\text { flexion }\end{array}$ & $\begin{array}{c}\text { maximum } \\
\text { extension }\end{array}$ & $\begin{array}{c}\text { ball } \\
\text { release }\end{array}$ & extension \\
\hline${ }^{1}$ normal & $61(1.3)$ & $176(1.1)$ & $175(0.6)$ & $198(0.2)$ & $187(0.9)$ & $5(0.6)$ \\
${ }^{1}$ yorker & $61(0.7)$ & $176(0.7)$ & $174(0.7)$ & $200(0.9)$ & $188(0.6)$ & $6(0.7)$ \\
${ }^{1}$ slower & $48(1.1)$ & $182(0.7)$ & $182(0.6)$ & $196(0.2)$ & $190(1.0)$ & $0(0.0)$ \\
${ }^{2}$ normal & $63(1.1)$ & $175(1.8)$ & $175(1.8)$ & $196(1.0)$ & $183(1.1)$ & $5(1.8)$ \\
${ }^{2}$ yorker & $62(1.0)$ & $177(1.2)$ & $177(1.2)$ & $196(0.6)$ & $184(0.7)$ & $3(1.2)$ \\
${ }^{2}$ bouncer & $65(1.6)$ & $176(2.4)$ & $176(2.3)$ & $195(0.9)$ & $182(0.9)$ & $4(2.3)$ \\
${ }^{2}$ normal ${ }^{*}$ & $62(2.0)$ & $177(1.8)$ & $176(1.8)$ & $196(0.7)$ & $183(1.8)$ & $4(1.8)$ \\
\hline
\end{tabular}

* indicates normal deliveries with 2007 bowling action. Elbow extension = most extended elbow extension angle (up to $180^{\circ}$ ) minus the preceding minimum elbow extension angle

Table 4. Elbow abduction angles during the bowling trials, ${ }^{1} 2007$ data, ${ }^{2} 2009$ data; mean (sd)

\begin{tabular}{lcccc}
\hline \multicolumn{4}{c}{ elbow abduction angle $\left(^{\circ}\right)$} \\
\hline $\begin{array}{c}\text { upper arm } \\
\text { horizontal }\end{array}$ & $\begin{array}{c}\text { minimum } \\
\text { abduction }\end{array}$ & $\begin{array}{c}\text { maximum } \\
\text { abduction }\end{array}$ & $\begin{array}{c}\text { ball } \\
\text { release }\end{array}$ \\
\hline${ }^{1}$ normal & $11(0.3)$ & $11(0.3)$ & $24(0.6)$ & $15(0.5)$ \\
${ }^{1}$ yorker & $11(0.4)$ & $11(0.4)$ & $24(0.3)$ & $15(0.5)$ \\
${ }^{1}$ slower & $15(0.6)$ & $14(0.5)$ & $22(0.8)$ & $17(0.4)$ \\
${ }^{2}$ normal & $7(0.8)$ & $6(0.7)$ & $27(0.4)$ & $16(0.5)$ \\
${ }^{2}$ yorker & $7(0.9)$ & $7(0.7)$ & $27(0.5)$ & $16(0.7)$ \\
${ }^{2}$ bouncel & $7(0.6)$ & $7(0.6)$ & $28(0.3)$ & $16(0.3)$ \\
${ }^{2}$ normal ${ }^{*}$ & $6(0.9)$ & $6(0.9)$ & $28(0.3)$ & $16(0.2)$ \\
\hline
\end{tabular}

* indicates normal deliveries with 2007 bowling action

Table 5. Comparison of elbow extension angles $\left(^{\circ}\right)$ at ball release calculated from the markers and measured from high speed video (2009 data)

\begin{tabular}{lccc}
\hline delivery type & trial & markers & high speed \\
\hline normal & 1 & 183 & 186 \\
& 2 & 183 & 185 \\
bouncer & 3 & 184 & 184 \\
& 1 & 182 & 180 \\
& 2 & 183 & 183 \\
yorker & 3 & 182 & 181 \\
& 1 & 185 & 183 \\
& 2 & 185 & 183 \\
normal $^{*}$ & 3 & 184 & 185 \\
& 1 & 183 & 184 \\
& 2 & 181 & 183 \\
& 3 & 184 & 182 \\
\hline
\end{tabular}

* indicates normal deliveries with 2007 bowling action 

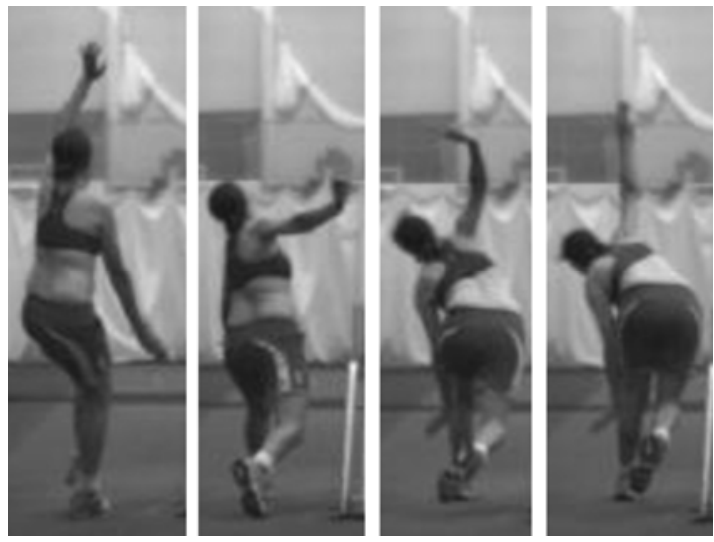

Figure 5. High speed video images for a typical normal length delivery.

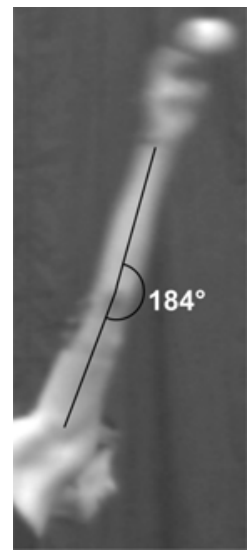

Figure 7. Side camera view at ball release for a typical trial with $4^{\circ}$ of elbow hyperextension (measured from high speed video still) compared with $3^{\circ}$ of elbow hyperextension calculated from the marker locations.
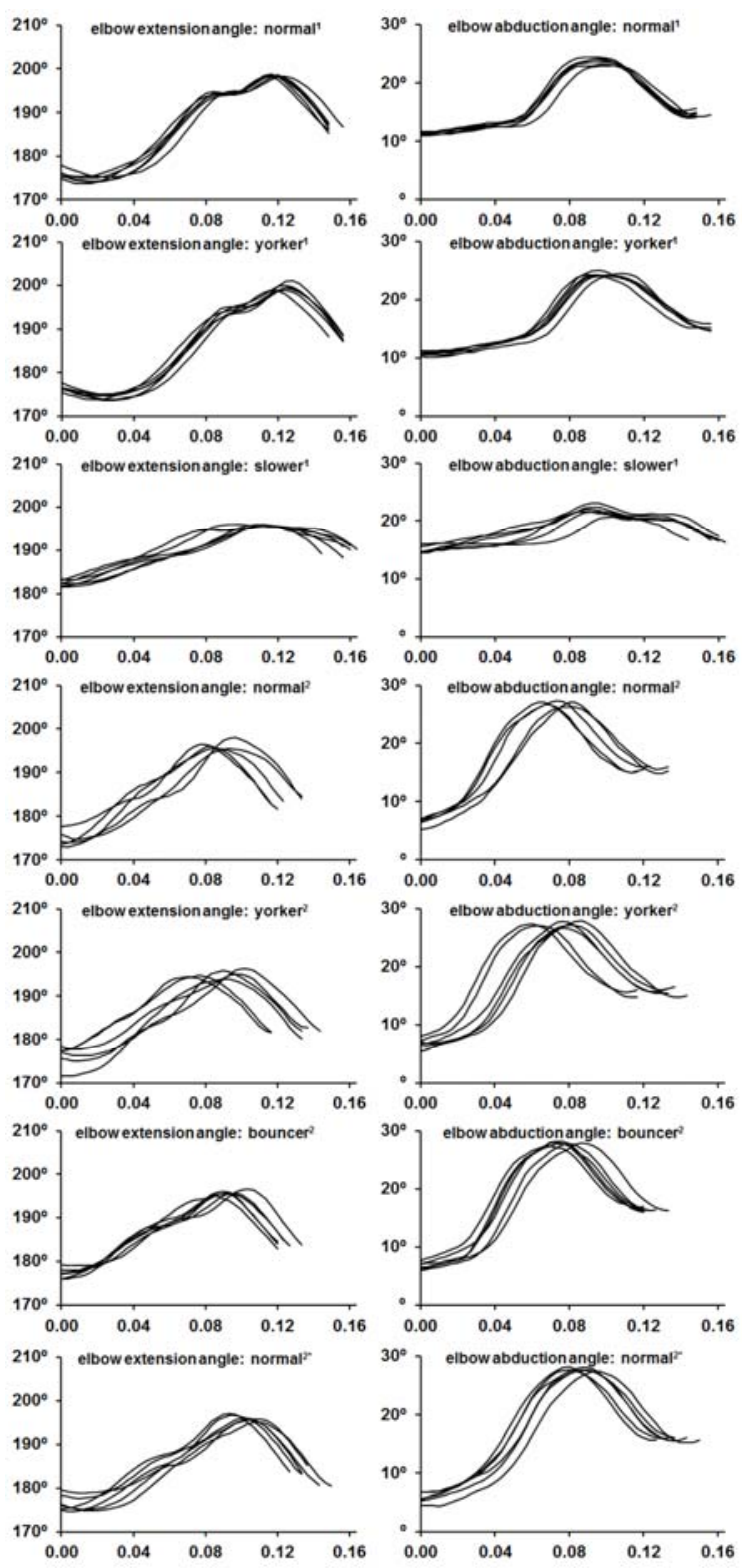

Figure 6. Elbow extension angle time histories and elbow abduction angle time histories for all recorded trials over the period upper arm horizontal to ball release, ${ }^{1} 2007$ data, ${ }^{2} 2009$ data.

Identifying the instants of upper arm horizontal and ball release to the nearest image resulted in the elbow being up to $11 \mathrm{~mm}$ above the level of the upper arm endpoint for upper arm horizontal while at ball release the distance between the ball marker and the wrist joint centre was up to $38 \mathrm{~mm}$ greater than the distance determined from the static ball release calibration trial. Using linear interpolation to determine between frames the instant of upper arm horizontal for each trial made less than $0.1^{\circ}$ difference to the calculated elbow extension angles. Using linear interpolation to determine the instant of ball release for each trial (ball to wrist joint 
centre distance equal to that measured during a static calibration trial) made up to $2^{\circ}$ difference to the calculated elbow extension angle at ball release but no difference to the calculated elbow extension due to Miss Gunn's particular bowling action. Filtering the data for a typical trial demonstrated obvious over-smoothing of the elbow extension angle time history when a $10 \mathrm{~Hz}$ or a $20 \mathrm{~Hz}$ cut-off frequency was used (Figure $8 \mathrm{a}$ and $8 \mathrm{~b}$ ), while filtering at $30 \mathrm{~Hz}$ or $40 \mathrm{~Hz}$ resulted in less than $1^{\circ}$ difference from the unfiltered elbow extension angles (Figure $8 \mathrm{c}$ and $8 \mathrm{~d}$ ).

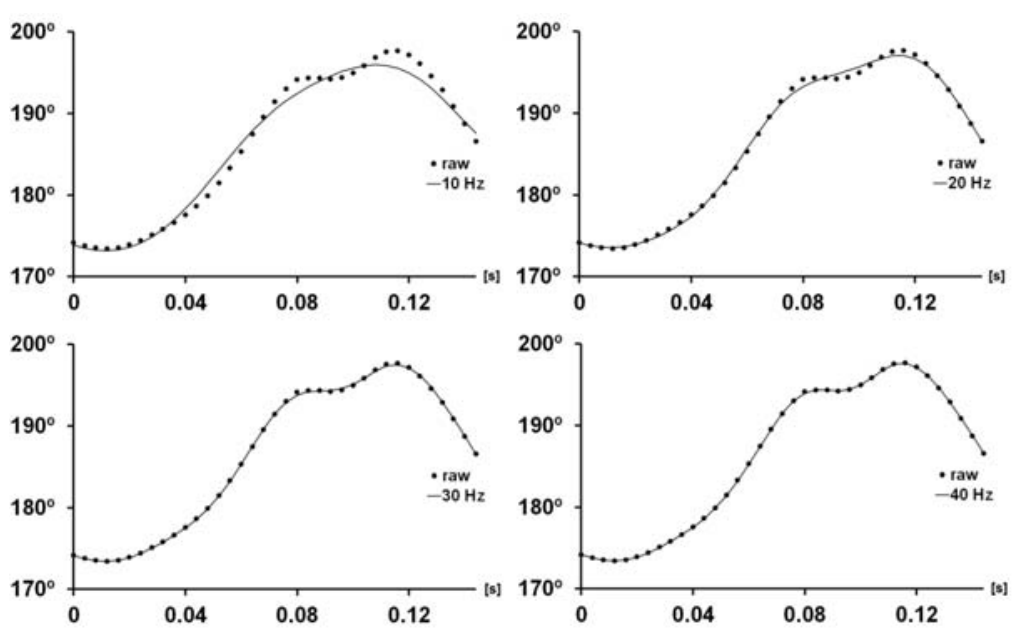

Figure 8. The effect of smoothing the displacement data at $10 \mathrm{~Hz}, 20 \mathrm{~Hz}, 30 \mathrm{~Hz}$ and $40 \mathrm{~Hz}$ on the elbow extension angle time history of a typical delivery by the subject between the instants of upper arm horizontal and ball release.

\section{Discussion}

This study has described a method appropriate for determining the amount of elbow extension and elbow abduction for a bowling delivery. Differences of up to $1^{\circ}$ for elbow extension and up to $2^{\circ}$ for elbow abduction were found when angles calculated from the marker set for static straight arm trials were compared with measurements taken by a chartered sports physiotherapist. In addition comparison of elbow extension angles at ball release calculated from the markers during bowling trials with those measured from high speed video also showed good agreement with mean differences of $0^{\circ} \pm 2^{\circ}$. These results indicate that the method produces results that correspond to independent visual measurements. It is clear from the analysis that Miss Gunn's bowling action is well within the allowed $15^{\circ}$ of elbow extension between upper arm horizontal and ball release with considerable amounts of elbow hyperextension and elbow abduction occurring (Table 3). Between the two data collection sessions Miss Gunn attempted to modify her bowling action as it was thought that this would be helpful and reduce the levels of extension. This was clearly unnecessary and it would appear that the efforts made to modify Miss Gunn's bowling action (2009 data) may have had a detrimental effect as Miss Gunn's 2009 bowling data was not as consistent as the corresponding 2007 bowling data (Figure $6)$.

The choice of axes from which elbow angles are calculated needs to correspond to the visual judgements made by umpires and so the use of midlines of the upper arm and lower arm is dictated by this requirement. Such a choice of axes needs to be made whether (a) these axes are calculated directly from a marker 
system as in this study or (b) a marker system based on minimal marker movement is used to determine technical axes and then a conversion is made to the above anatomical system (Kontaxis et al., 2009). The use of anatomical axes based upon the underlying bony structure may be appropriate for clinical applications but is inappropriate for bowling due to the discrepancy in what constitutes a "straight" arm or "upper arm horizontal" compared with an umpire's visual judgement. Since the aim was to determine the elbow angles from the orientations of the midlines of the upper arm and lower arm, the locations of the centres of the gleno-humeral joint, elbow joint and wrist joint are irrelevant. For this reason an upper arm endpoint lying on the midline of the upper arm, a lower arm endpoint lying on the midline of the lower arm, and an "elbow centre" lying on both midlines were used rather than shoulder, wrist and elbow joint centres based on relative movements of the underlying bones as used by others (Lloyd et al., 2000; Alderson et al., 2004; Elliott and Alderson, 2007; Elliott et al., 2005; Elliott et al., 2007; Campbell et al., 2009a, 2009b).

Marker placement was dictated by the need to define the midlines of upper and lower arm segments and to minimise the effects of skin and soft tissue movement. The pair of markers at the shoulder were attached with the arm overhead to overcome the problem of skin movement at the shoulder when the arm moves overhead while bowling. The pair of markers around the elbow are likely to have less movement artefacts arising from skin movement during internal / external rotation of the upper arm than small triads placed over soft tissue as advocated by Lloyd et al. (2000) and Alderson et al. (2004). Furthermore since the joint angle changes at the elbow are relatively small the issue of skin movement as the joint angle changes (Cappozo et al., 1996) will be small. Using a small marker triad and extrapolating to calculate joint centres and axes has the inherent problem that any tracking errors are magnified when the joint centres and axes are reconstructed. In addition, these other methods may be prone to soft tissue artefacts which result in errors in calculated joint centres and axes due to the triads of markers moving relative to the arm (Zhang et al., 2011). Indeed Cutti et al. (2006) state that "soft tissue artefact is the dominant error source for upper extremity motion analyses that use skin-mounted markers" while Roosen et al. (2009) state that for athletic human movement "determining the joint centre of the shoulder or elbow with a triad of markers per segment with an accuracy greater than $20 \mathrm{~mm}$ is unlikely". The method presented here effectively uses a large triad comprising upper arm endpoint and elbow markers to define an upper arm reference frame rather than using a small marker triad. In the future it would be appropriate to compare the amount of movement artefact from using three pairs of markers with a cluster based approach.

A functional elbow axis was determined in this study from an "elbow flexion calibration trial" with the arm overhead. The functional elbow axis is held in the coordinate system of the upper arm as recommended by Wu et al. (2005) and elbow extension is the rotation about this axis. A measure of the accuracy of this procedure is the standard deviation of the abduction angle during the elbow flexion calibration trials since the abduction angle (or carry angle) should remain constant for a fixed hinge joint. Since the standard deviation from the two trials were $0.7^{\circ}$ and $1.1^{\circ}$ it may be concluded that the functional elbow axis procedure produces a good fit to the data despite only using data from two extension angles (near $180^{\circ}$ and $90^{\circ}$ ) to define the axis. A functional axis has the advantage of being specific to the individual and not particularly dependent upon on marker placement (Leardini et al., 1999). In the 
future it would be appropriate to compare the effect of elbow axis definition on the calculated elbow extension angles.

The marker based elbow extension angles were within $2^{\circ}$ of independent measures made from the static trials and bowling trials. In addition during the bowling trials the arm was forced into $15^{\circ}$ to $20^{\circ}$ of hyperextension (Table 3 , Figure 6 ) with the measurements consistent with a static hyperextension angle of $14^{\circ}$ and high speed video recordings. Previous methods (Lloyd et al., 2000; Alderson et al., 2004; Elliott et al., 2005; Elliott et al., 2007) have not compared calculated angles from a bowling trial to any estimates obtained from video and have assumed that their calculated angles are appropriate.

Identifying upper arm horizontal and ball release more exactly for Miss Gunn using linear interpolation made little difference to the calculated levels of elbow extension, but for some bowlers it could potentially make much larger differences. Furthermore the current protocols specify an arbitrary sampling frequency of $250 \mathrm{~Hz}$, despite modern motion analysis systems being able to capture at $480 \mathrm{~Hz}$ with full resolution. It would seem appropriate that the highest recording frequency where markers can be $100 \%$ tracked should be used, with linear interpolation between images used to identify the instants of upper arm horizontal and ball release. Although the current protocols specify that raw displacement data should be smoothed, this study has shown that this is not necessary since a sensible level of smoothing made less than $1^{\circ}$ difference to the calculated elbow extension angles (Figure 8). The disadvantage of smoothing the displacement data is that it adds extra complexity and a decision needs to be made on the amount of smoothing to be used: with increased smoothing more of the peaks and troughs are truncated and as a consequence the calculated elbow extension could be reduced erroneously.

The method that has been developed is specifically for the determination of the amount of elbow extension/hyperextension between upper arm horizontal and ball release for cricket bowling. The method has been designed with a minimal marker set so as to allow the bowler to bowl as naturally as possible in a laboratory environment and an objective method is used to ensure that a straight arm position is identified with confidence for an individual bowler. In this study a number of issues have been addressed that the current ICC protocols do not consider and the method has been demonstrated to give good reproducible results for a bowler with an unusual bowling action across two bowling sessions 18 months apart. In the future it would be appropriate to compare angles calculated using current ICC protocols with those obtained from the method presented in this paper.

\section{References}

Aginsky, K.D., \& Noakes, T.D. (2010). Why it is difficult to detect an illegally bowled cricket delivery with either the naked eye or usual two-dimensional video analysis, British Journal of Sports Medicine, 44, 420-425.

Alderson, J., Elliott, B., Reid, S., Portus, M., \& Lloyd, D. (2004). Vicon assists in unravelling illegal bowling dilemma. www.viconstandard.org. Accessed 17/11/2004.

Bartlett, R.M. (2003). The science and medicine of cricket: an overview and update, Journal of Sports Sciences, 21, 733-752.

Campbell, A.C., Alderson, J.A., Lloyd, D.G., \& Elliott, B.C. (2009a). Effects of different technical coordinate system definitions on the three dimensional 
representation of the glenohumeral joint centre. Medical \& Biological Engineering \& Computing, 47, 543-550.

Campbell, A.C., Lloyd, D.G., Alderson, J.A., \& Elliott, B.C. (2009b). MRI development and validation of two new predictive methods of glenohumeral joint centre location identification and comparison with established techniques. Journal of Biomechanics, 42, 1527-1532.

Cappozzo, A., Catani, F., Leardini, A., Benedetti, M.G., \& Della Croce, U. (1996). Position and orientation in space of bones during movement: Experimental artifacts. Clinical Biomechanics, 11, 90-100.

Chin, A.W., Elliott, B.C., Alderson, J.A., Lloyd, D.G., \& Foster, D.H. (2009). The offbreak and "doosra": Kinematic variations of elite and sub-elite bowlers in creating ball spin in cricket bowling. Sports Biomechanics, 8, 187-198.

Chin, A.W., Lloyd, D.G., Alderson, J.A., Elliott, B.C., \& Mills, P. (2010). A markerbased mean finite helical axis model to determine elbow rotation axes and kinematics in vivo. Journal of Applied Biomechanics, 26, 305-315.

Cutti, A.G., Cappello, A., \& Davalli, A. (2006). In vivo validation of a new technique that compensates for soft tissue artefact in the upper-arm: Preliminary results. Clinical Biomechanics, 21, S13-S19.

Elliott, B.C., \& Alderson, J.A. (2007). Laboratory versus field testing in cricket bowling: A review of current and past practice in modelling techniques. Sports Biomechanics, 6, 99-108.

Elliott, B.C., Alderson, J.A., \& Denver, E.R. (2007). System and modelling errors in motion analysis: Implications for the measurement of the elbow angle in cricket bowling. Journal of Biomechanics, 40, 2679-2685.

Elliott, B.C., Reid, S., Alderson, J.A., \& Lloyd, D.G. (2005). The Muralitharan Doosra: technology and the law in cricket. Sport Health, 23, 3, 13-15.

International Cricket Council Website. (2009). ICC regulations for the review of bowlers reported with suspected illegal bowling actions. Accessed 05/08/2011. http://static.icccricket.yahoo.net/ugc/documents/DOC 34EE3BB667212C9A0C32EFBFF19E2 F2F 1263367785914 117.pdf.

Kontaxis, A., Cutti, A.G., Johnson, G.R., \& Veeger, H.E.J. (2009). A framework for the definition of standardized protocols for measuring upper-extremity kinematics. Clinical Biomechanics, 24, 246-253.

Leardini, A., Cappozzo, A., Catani, F., Toksvig-Larsen, S., Petitto, A., Sforza, V., Cassanelli, G., \& Giannini, S. (1999). Validation of a functional method for the estimation of hip joint centre location. Journal of Biomechanics, 32, 99-103.

Lloyd, D.G., Alderson, J., \& Elliott, B.C. (2000). An upper limb kinematic model for the examination of cricket bowling: A case study of Muttiah Muralitharan. Journal of Sports Science, 18, 975-982.

Portus, M.R., Rosemond, C.D., \& Rath, D.A. (2006). Fast bowling arm actions and the illegal delivery law in men's high performance cricket matches, Sports Biomechanics, 5, 215-230. 
Roosen, A., Pain, M.T.G., \& Begon, M. (2009). Limitations of functionally determined joint centres for analysis of athletic human movement: A case study of the upper limb. Journal of Applied Biomechanics, 25, 281-292.

Wu, G., van der Helm, F.C.T., Veeger, H.E.J., Makhsous, M., van Roy, P., Anglin, C., Nagels, J., Karduna, R.K., McQuade, K., Wang, X., Werner, F.W., \& Buchholz, B. (2005). ISB recommendation on definitions of joint coordinate systems of various joints for the reporting of human joint motion - Part II: shoulder, elbow, wrist and hand. Journal of Biomechanics, 38, 981-92.

Zhang, Y., Lloyd, D.G., Campbell, A.C., \& Alderson, J.A. (2011). Can the Effect of Soft Tissue Artifact Be Eliminated in Upper-Arm Internal-External Rotation? Journal of Applied Biomechanics, 27, 258-265. 